\title{
Numerical simulation on the effectiveness of using viscoelastic materials to mitigate seismic induced vibrations of above-ground pipelines
}

\author{
Kaiming $\mathrm{Bi}^{*}$, Hong Hao \\ Centre for Infrastructure Monitoring and Protection, School of Civil and Mechanical \\ Engineering, Curtin University, Kent Street, Bentley WA 6102, Australia
}

\begin{abstract}
Pipeline systems are commonly used to transport oil, natural gas, water, sewage and other materials. They are normally regarded as important lifeline structures. Ensuring the safety of these pipeline systems is crucial to the economy and environment. There are many reasons that may result in the damages to pipelines and these damages are often associated with pipeline vibrations. Therefore it is important to control pipeline vibrations to reduce the possibility of catastrophic damages. This paper carries out numerical investigations on the effectiveness of using viscoelastic materials to mitigate the seismic induced vibrations of above-ground pipelines. The numerical analyses are carried out by using the commercial software package ANSYS. The numerical model of the viscoelastic material is firstly calibrated based on the experimental data obtained from vibration tests of a $1.6 \mathrm{~m}$ long tubular sandwich structure. The calibrated material model is then applied to the above-ground pipeline system. The effectiveness of using viscoelastic materials as the seismic vibration control solution is investigated. The influences of various parameters, including the constraining arrangement scenarios, the constraining length and angle, the thicknesses of the viscoelastic material and constraining layer are discussed in detail. The influence of earthquake frequency content is discussed as well. Numerical results show that with properly selected viscoelastic materials and constraining layers, the proposed method can be used to effectively mitigate seismic induced vibrations of above-ground pipelines.
\end{abstract}

Keywords: viscoelastic material, above ground pipeline, vibration control, numerical simulation

*Corresponding author. Tel: +61 89266 5139; fax: +61 892662681

E-mail address: kaiming.bi@curtin.edu.au (K. Bi) 


\section{Introduction}

Pipeline systems are commonly used to transport oil, natural gas, water, sewage and other materials. They are normally regarded as important lifeline structures. Ensuring the safety of these pipeline systems is crucial to the economy and environment. There are many reasons that may result in the damages of pipelines. These include possible corrosion and fatigue damages after the pipeline is in service for a number of years [1], damage owing to large bending deformation and excessive stresses in the pipe wall induced by large external loadings [2-4] and damage related to lateral buckling, upheaval buckling or propagation buckling [5]. Often damages may also be associated with pipeline vibrations. For example, vortex-induced vibrations (VIV) of subsea pipelines [6], vibrations caused by earthquake excitations in seismic active zones [7] or vibrations induced by strong winds [8]. These dynamic loadings may induce excessive stresses in the pipe structure and lead to damage. Even if the vibration level is not large enough to cause overstress in the pipeline structure, relatively large continuous vibration such as VIV certainly reduces the fatigue life of the pipe. Therefore, it is important to control pipeline vibrations to reduce the possibility of catastrophic damage.

When the soil deformations produced by the buried pipelines are unacceptably large, the above-ground pipeline can be an option to carry fluid or gas [9]. These pipelines are generally supported along their length by discrete concrete blocks. The suspended spans may undergo excessive vibrations during a severe earthquake, which in turn can result in damages to the pipelines. Previous studies on the seismic responses of above-ground pipelines are surprisingly rare. Anderson and Johnston [10] investigated the dynamic behaviour of aboveground oil pipelines. These pipelines are allowed to slide back and forth on intermediate supports during strong earthquakes. The sliding is restrained by friction between the pipe and the top of the support. The effect of this non-linear friction on both the static and dynamic stresses in the pipe was discussed. Powell [9] developed a procedure to compute the seismic response of above-ground, cross-country pipelines. The procedure can account for the effects of initial static loads, slipping of the pipe on its supports and out-of-phase ground motions at different supports along the pipe. Soliman and Datta [11] carried out parametric studies on the seismic responses of overground pipelines to multi-component random ground motions. The mean square responses of the pipelines were obtained by frequency domain spectral analysis. Lanzano et al. [12] presented a large database of earthquake-induced damage for steel and non-steel pipelines. 
To mitigate the excessive structural vibrations induced by various sources, three types of control strategies, i.e., active, semi-active and passive controls, can be used in the structural vibration resistance design [13]. Considerable attention has been paid to research and development of structural control devices, with particular emphasis on the mitigation of wind and seismic induced responses of buildings and bridges. Studies on pipeline vibration control are relatively less and they are mainly focused on the passive control of VIV [6]. More recently, tuned mass dampers (TMD) were introduced to control wind [8] or vortex-induced [14] vibrations of pipelines. However, it has been observed that most of these methods are difficult to achieve a good balance between performance, cost and simplicity [6].

Constrained viscoelastic layers have been widely used to reduce excessive vibrations of engineering structures due to its effectiveness and simplicity (e.g. [15-18]). Normally a layer or multiple layers of viscoelastic materials (VEM) and a constraining layer (CL) are added to the original structure. The shear deformation of the VEM can obviously increase the damping of the original structure which in turn reduces its vibration. Extensive research efforts have been made to study the vibration characteristics of beam and plate structures with constrained damping layer after the pioneering work done by Kerwin [19] and Ross et al. [20]. For the vibration and damping characteristics of cylindrical shells with constrained damping layer(s), the investigations are relatively less and the natural frequencies and damping of the constrained shell were generally derived based on the finite element method. For example, Chen and Huang [21] presented a mathematical model for a cylindrical shell with partially constrained layer damping treatment. A thin shell theory in conjunction with the DonnellMushtari-Vlasov assumptions is employed to yield the model. Wang and Chen [22] derived the equations of motion for the composite system based on a discrete layer theory. Many lengthy formulas were included in these studies, which impedes the application of these theories by researchers and especially engineers. A more readily applicable method, e.g. the numerical simulation method presented in this study, is deemed necessary.

This paper investigates the effectiveness of using constrained VEM layers to mitigate seismic induced vibrations of above-ground pipelines. This idea originates from the recent work done by Borges et al. [16], in which they proposed and investigated a concept aimed at suppressing vibrations in steel catenary risers by the use of viscoelastic sandwich layers. A series of experimental studies were carried out to find out the frequencies and damping of different vibration modes of the riser equipped with different (eleven) scenarios of VEM. Instead of performing experimental studies, numerical simulations are carried out in the 
present study to investigate the effectiveness of using viscoelastic materials as the seismic vibration control solution to above-ground pipelines by using the commercial software package ANSYS [23]. The numerical model of the viscoelastic material is calibrated based on the experimental data obtained from testing a $1.6 \mathrm{~m}$ long tubular sandwich structure [16] in Section 2. The calibrated material model is then applied to the above-ground pipeline system. The effectiveness of using constrained VEM as the seismic vibration control solution is investigated. The influences of various parameters, including the constraining arrangements, the constraining length and angle, the thicknesses of the VEM and CL are discussed in detail. The influence of earthquake frequency content is discussed as well.

\section{Numerical model calibration}

\subsection{Tested original and sandwich tubes}

Borges et al. [16] carried out a series of experimental studies to identify the modal parameters (vibration frequencies and damping) of the original structure and structures assembled with different VEMs and CLs. The original structure consists of a brass beam with tubular cross section that is cantilevered at one end and free at the other. The length of the original structure is $1.6 \mathrm{~m}$. To increase the damping of the original structure, viscoelastic layers and its associated brass constraining layers are assembled. The VEM used is the selfadhesive double face tape under code VHB4955, manufactured by $3 \mathrm{M}^{\circledR}$. The viscoelastic and constraining layers are designed to be free at the both ends. Fig. 1 shows the cross section of the sandwich beam and Table 1 presents the geometric properties of the tube layers.

For the brass original tube and CLs, the Young's modulus is $E_{b}=121.8 \mathrm{GPa}$ and the density is $\rho_{b}=8770 \mathrm{~kg} / \mathrm{m}^{3}$. For a linear, homogeneous and isotropic VEM, the complex shear modulus can be expressed in the frequency domain as

$$
G^{*}(\omega)=G(\omega)[1+i \beta(\omega)]
$$

where $G(\omega)$ is the storage modulus, $\beta(\omega)$ is the dissipation loss factor, $\omega$ is the circular frequency in rad/s and $i=\sqrt{-1}$ is the imaginary number. $G(\omega)$ and $\beta(\omega)$ can be obtained by using one of the following two types of tests, i.e., the direct measurements using a Dynamic Mechanical Analyser (DMA) [15, 24] or back calculation from experimental results performed on the sandwich structure [15]. For the VEM used in the present study, the following parameters are identified: Young's modulus $E_{v}=6.88 \mathrm{MPa}$, density $\rho_{v}=795 \mathrm{~kg} / \mathrm{m}^{3}$ 
Poisson's ratio $v=0.49$ and dissipation loss factor $\beta=0.75$. The shear modulus is thus $G=E /[2(1+v)]=2.31 \mathrm{MPa}$. These parameters are adopted from Stutz et al. [25], in which the same VEM was used.

It can be seen from Fig. 1 that the original tube was not fully covered by the VEMs and CLs, a gap was designed between different faces of cover layers. The angle of the gap was not mentioned in [16]. Based on the provided figure (Fig. 8 in [16]), the angle is estimated to be $18^{\circ}$ and used in the present study, the angle of each constraining layer is thus $72^{\circ}$ as shown in Fig. 1.

\subsection{Finite element modelling}

The finite element software package ANSYS is used in the present study to carry out the analyses. The original tube, VEMs and CLs are all modelled with solid element SOLID186. This higher order element exhibits quadratic displacement behaviour and it is defined by 20 nodes having three degrees of freedom per node: translations in the $\mathrm{x}, \mathrm{y}$ and $\mathrm{z}$ directions. Moreover, this element type supports viscoelasticity. In the numerical model, the circumference of the original tube is divided into 40 elements. In the radial direction, the original tube and CLs are modelled by one element respectively while the VEMs are divided into two. In the longitudinal direction, the element size is $16 \mathrm{~mm}$. The VEMs are rigidly connected to the original tube and CLs, namely the VEMs share nodes with the original tube and CLs. The cross section of the original tube is relatively small compared to its length, plotting the whole FE model will make the figure not clear, Fig.2 shows part of the FE model of the original tube with faces 1 and 2 constrained by VEMs and CLs.

The constraining layers are assumed to be linear elastic, while the VEM is assumed to be hyperelastic [15]. The damping is modelled in ANSYS for each material as a constant stiffness multiplier (DAMP in ANSYS), which can be calculated from [15]:

$$
\alpha_{2}=\frac{\zeta}{\pi f}
$$

where $\alpha_{2}$ is the stiffness multiplier, $f$ is the fundamental vibration frequency of the structure, which can be obtained by carrying out an eigenvalue analysis, $\zeta$ is the damping ratio of the material. For the viscoelastic material $\zeta$ is related to the dissipation loss factor $\beta$ and can be estimated as $\zeta=\beta / 2$ [26]. For the original tube, the damping ratio is $0.05 \%$ based on the test results [16]. 


\subsection{Numerical and experimental results}

Three different types of structures were tested by Borges et al. [16], i.e. the original tube, the tube with faces 1 and 2 (see Fig. 1) constrained with VEMs and CLs, and the tube with all faces constrained. The modal parameters in directions 1 and 2 (Fig. 1) were experimentally identified. All these three different cases are numerically simulated and compared with the experimental data. To identify the modal parameters in these two different directions, a $5 \mathrm{~mm}$ initial displacement is introduced in directions 1 and 2 respectively at the free end of the system and then released suddenly to simulate a free vibration test. The free vibration responses are calculated and Fig. 3 shows the displacement time histories at the free end of the tube with faces 1 and 2 constrained. For the original tube and the tube with all faces constrained, the free vibration displacement time histories are not shown for conciseness. The free vibration responses are then used as input to identify the modal parameter.

There are many methods, e.g. the Short Time Fourier Transform (STFT) method, the wavelet transform method, etc, available to identify the modal parameters once we have the free vibration data of the system. In the present study, the wavelet transform method proposed by Ruzzene et al. [27] is adopted. This method is drawn upon the unique characteristics of Morlet wavelets, and the modal parameters are identified from the modulus and phase angle of the wavelet transform of the free vibration data. For more detailed information regarding this method, readers can refer to [27].

Tables 2 to 4 tabulate the identified modal parameters of the first two modes at directions 1 and 2 of the three different types of structures based on the numerical results and the corresponding values obtained from the tests. The differences between the numerical and experimental results, which are calculated from $\left(R_{N}-R_{E}\right) / R_{E}$, are also tabulated, where $R_{N}$ and $R_{E}$ represent the numerical and experimental results respectively. It is noted that the sampling frequency of the numerical results is $500 \mathrm{~Hz}$ in the present study. As shown in Tables 2 to 4, the modal parameters identified from the numerical results coincide well with those from the experimental tests. The differences between the numerical and experimental results are mostly within $15 \%$. Large differences occur at the damping ratio of the original tube. This is because, as can be seen from Table 2 that the absolute value of the experimental results are quite small (0.05\% and $0.03 \%)$, a slight deviation from the experimental results can lead to a large difference. The numerical simulation adopted in the present paper is therefore believed able to realistically model the VEM and the sandwich structure. It also can be seen from the 
tables that the constrained VEM can significantly increase the damping ratio of the structure. It thus has the potential to reduce the vibration of the original structure.

It should be noted that the tested original tube presented some imperfections [16]. The natural frequencies in the two different directions obtained from the tested data are slightly different as shown in Tables 2 and 4 . In the numerical simulation, these imperfections are not considered, and the corresponding values in these two different directions are thus the same for the symmetrical cross sections, i.e., in the case of original tube (Table 2) and the tube with all faces constrained (Table 4).

\section{Above-ground pipelines}

\subsection{Pipeline details}

Fig. 4 shows a typical above-ground pipeline supported on discrete supports at equal intervals. The pipeline is made of steel and the length of each span is $16 \mathrm{~m}$. The outer diameter of the pipe cross section is $0.35 \mathrm{~m}$ and the thickness is $3 \mathrm{~mm}$. The pipeline may undergo violent vibrations under severe earthquakes. To mitigate these adverse vibrations, VEM layers and CLs are proposed to be assembled on the surface of the original pipeline. In a real earthquake, three dimensional ground excitations are inevitable. To demonstrate the effectiveness of the proposed method, only the transverse earthquake loading is, however, considered in the present study. The VEMs and CLs are only assembled in the transverse direction (x direction as shown in Fig. 5) of the pipe.

\subsection{Seismic ground motion}

The pipeline is located on a flat-lying soil site as shown in Fig. 6. One single layer of soil rests on the base rock. The parameters of the soil layer and base rock are included in the figure, where $\rho, G, \zeta, v$ and $h$ represent the density, shear modulus, damping ratio, Poisson's ratio and thickness respectively. The lower cases $s$ and $b$ represent the soil layer and base rock. In the present study, the base rock motion is assumed to consist of out-of-plane $S H$ wave and it is represented by a filtered Tajimi-Kanai power spectral density function as [28]

$$
S_{g}(\omega)=\frac{\omega^{4}}{\left(\omega_{f}^{2}-\omega^{2}\right)^{2}+\left(2 \omega_{f} \omega \xi_{f}\right)^{2}} \frac{1+4 \xi_{g}^{2} \omega_{g}^{2} \omega^{2}}{\left(\omega_{g}^{2}-\omega^{2}\right)^{2}+4 \xi_{g}^{2} \omega_{g}^{2} \omega^{2}} \Gamma
$$


where $\omega_{g}$ and $\xi_{g}$ are the central frequency and damping ratio of the Tajimi-Kanai power spectral density function, $\omega_{f}$ and $\xi_{f}$ are the corresponding central frequency and damping ratio of the high pass filter function. $\Gamma$ is a scaling factor depending on the ground motion intensity. The parameters for the transverse motion are assumed as $\omega_{g}=10 \pi \mathrm{rad} / \mathrm{s}, \xi_{g}=0.6$, $\omega_{f}=0.5 \pi, \xi_{f}=0.6$ and $\Gamma=0.0212 \mathrm{~m}^{2} / \mathrm{s}^{3}$. These parameters correspond to a ground motion time history with duration $T=16 \mathrm{~s}$ and PGA of $0.5 \mathrm{~g}$ based on the standard random vibration method [29].

The base rock motion can be further filtered and amplified by the soil layer. The transverse earthquake loading on the ground surface (x direction in Figs. 5 and 6) can be simulated based on the combined spectral representation method and one-dimensional wave propagation method [30]. Fig. 7 shows the simulated transverse acceleration time history. It is worth to note that in the simulation, the sampling frequency and the upper cut-off frequency are set to be 100 and $25 \mathrm{~Hz}$ respectively. Fig. 8 shows the comparison of the simulated power spectral density (PSD) with the theoretical value which is derived based on the onedimensional wave propagation theory [31]. Good agreements are observed.

\subsection{Numerical model}

The viscoelastic material VHB4955 manufactured by $3 \mathrm{M}^{\circledR}$ calibrated in Section 2 is used again to increase the damping of the original pipeline. The sandwich pipeline is modelled the same way as the sandwich tube in Section 2, namely, SOLID186 elements are used to simulate the original pipeline, VEMs and CLs; VEMs are rigidly connected with original pipeline and CLs; the original pipeline is divided into 40 elements along its circumference and 100 elements in the longitudinal direction; in the radial direction, the original tube and CLs are modelled by one element respectively while the VEMs are divided into two; and the damping is modelled as a constant stiffness multiplier for each material.

The original pipe and the CLs are made of steel and the Young's modulus, density and Poisson's ratio are $210 \mathrm{GPa}, 7800 \mathrm{~kg} / \mathrm{m}^{3}$ and 0.3 respectively. Normally the pipe is not fully fixed to the supports, the transverse restraint provided by the support can be considered by a spring. The stiffness of the spring normally varies from $7.5 \times 10^{5} \mathrm{~N} / \mathrm{m}$ to $6 \times 10^{6} \mathrm{~N} / \mathrm{m}$ [10]. In the present study, the transverse restraints provided by the supports are modelled by the COMBIN14 elements, and its stiffness is $1.1644 \times 10^{6} \mathrm{~N} / \mathrm{m}$ [11]. In the vertical direction, the 
pipeline is assumed to be simply supported by the supports. The damping ratio of the original pipeline is assumed to be $1.2 \%$ in the present study.

Since it is impossible to model the whole length of a pipeline system, taking one span of the entire pipeline out for analysis is more practical. To simulate the restraining effects from adjacent spans to the single-span model, rotational springs are added at the both ends of the analysed span [32], and they are modelled by COMBIN14 elements again. The rotational spring stiffness is determined by performing a numerical convergence analysis. In which, the transient analyses of multi-span pipeline models are firstly performed. Fig. 9 shows that the displacement responses of the middle point of a seven-span and a nine-span model are almost the same when the multi-span models are subjected to the transverse earthquake loading as shown in Fig. 7. The dynamic response of the middle span in a seven-span model is thus used as the reference for determining the rotational spring stiffness. Based on the convergence analysis, a value of $1.465 \times 10^{5} \mathrm{Nm} / \mathrm{rad}$ is determined as the rotational spring stiffness. The displacement response at the middle point of the single-span model is also shown in Fig. 9, and good agreement is observed. Fig. 10 shows part of the single-span model, in which the whole span is assembled with VEMs and CLs. In the subsequent analysis, only the single-span model with the rotational spring at the both supports is analysed. This substantially reduces the computational time for the analysis.

\section{Numerical results}

This section carries out parametric studies on the effectiveness of using constrained VEMs to mitigate the seismic induced vibrations of above-ground pipelines. The influences of various parameters related to the VEMs and CLs on the vibration frequency and damping ratio of the pipeline system, as well as on the seismic responses are discussed in detail. The influence of earthquake frequency content is discussed as well. For comparison, the corresponding results from the original pipeline are also presented.

The acceleration time history shown in Fig. 7 is used as input in the transverse direction of the pipeline. The duration of the earthquake loading is $16 \mathrm{sec}$. In the numerical simulation, a $20 \mathrm{sec}$ response is calculated. In the first $16 \mathrm{sec}$, the pipeline system is subjected to the transverse earthquake loading (forced vibration), while it vibrates freely in the last $4 \mathrm{sec}$. The acceleration response in the free vibration phase is used to identify the modal parameters, i.e., natural frequency and damping ratio of the system, based on the wavelet transform method proposed by Ruzzene et al. [27] with a sampling frequency of $100 \mathrm{~Hz}$. Since the transverse 
input is considered in the present study, only the vibration frequency and damping ratio corresponds to the first transverse vibration mode are presented and discussed. For the original pipeline, the fundamental vibration mode is in the transverse direction with a frequency of $3.8369 \mathrm{~Hz}$ based on an eigenvalue analysis. By using the free vibration result, the identified frequency and damping ratio is $3.8556 \mathrm{~Hz}$ and $1.25 \%$ respectively, which are close to the vibration frequency obtained from the eigenvalue analysis and the assumed damping ratio of $1.2 \%$. All the modal parameters presented in the following sections are the identified values based on the single-span pipeline model.

\subsection{Influence of constraining arrangement scenarios}

Borges et al. [16] experimentally identified the vibration frequencies and damping of the original riser with the viscoelastic sandwich layers sequentially assembled in segments along the original structure. This segmented arrangement (Fig. 11(a)) as suggested in [16] is firstly investigated in the study. The length for each segment is $2 \mathrm{~m}$ and the spacing between adjacent segments is $1 \mathrm{~m} .1$, 3 and 5 constraining segments are considered as shown in Fig. 11(a). Another two arrangement scenarios, namely the compact configuration shown in Fig. 11(b)) and the monolithic configuration shown in Fig. 11(c), are also investigated. In the compact configuration, the constraining segments concentrate at the centre of the span and there is no gap between each segment. For the monolithic configuration, all the segments are rigidly connected together to form an integral constraining. In all these cases, the thickness of the VEM layers is $20 \mathrm{~mm}$ and the thickness of the CLs is $3 \mathrm{~mm}$. The constraining angle $\alpha$ as shown in Fig. 5 is $72^{\circ}$.

Fig. 12 shows the identified fundamental transverse vibration frequencies and the corresponding damping ratios of different constraining arrangement cases. The results obtained from the original pipeline (constraining length $\mathrm{L}=0 \mathrm{~m}$ ) are also plotted. As shown in Fig. 12(a), for the segmented and compact arrangements, increasing the number of constraining segments leads to the monotonous decreasing of vibration frequency of the system. For the monolithic arrangement, the vibration frequency decreases with the increasing of the constraining length if the constraining length is less than $6 \mathrm{~m}$. When the constraining length reaches $10 \mathrm{~m}$, the vibration frequency of the system is, however, larger than the pipeline with the constraining length of $6 \mathrm{~m}$. This is because the vibration frequency is determined by the mass and stiffness of the system. For the segmented and compact arrangements, the segments contribute relatively small to the stiffness of the system because 
of the short length of the segments ( $2 \mathrm{~m}$ in the present study). Increasing the segment numbers, however, obviously increases the mass of the system, which in turn results in the smaller vibration frequency. For the monolithic arrangement with long enough VEMs and CLs, the constraining layers will evidently increase the stiffness of the system as well, besides their contributions to the mass. When the contribution to the stiffness is larger than that to the mass, larger vibration frequency will be obtained. It also can be seen from Fig. 12(a) that the compact arrangement leads to smaller vibration frequency of the system compared to the segmented configuration. This is because the segments contribute more to the total mass of the system when they are more concentrated to the centre of the span.

Fig. 12(b) shows the influence of constraining arrangement scenarios on the damping ratio of the system. As can be seen from the figure, the segmented and compact arrangements only slightly increase the damping ratio of the system. For the original pipeline, the identified damping ratio is $1.25 \%$. When 2, 6 and $10 \mathrm{~m}$ constraining layers are assembled, the damping ratios are $1.43 \%, 1.67 \%$ and $1.70 \%$ respectively for the segmented arrangement. The corresponding values for the compact arrangement are $1.43 \%, 1.69 \%$ and $1.81 \%$. On the other hand, the increasing of damping to the system is quite obvious if the constraining layers are assembled monolithically when the length of the constraining layers is not too short. For example, the damping ratios reach 3.53\% and 4.78\% when the constraining lengths are 6 and $10 \mathrm{~m}$ respectively. This is because the high damping capacity of structure with constrained damping layer is mostly due to the shear deformation of the VEM [22]. With the same length of constraining layers, the VEMs and CLs undergo larger shear deformation during vibration when monolithic arrangement is considered and thus larger damping ratio is expected.

The constraining layers can significantly influence the seismic responses of the system. Fig. 13 shows the transverse displacement time histories at the middle span of the pipelines with different scenarios of constraining. Only the forced vibration responses are plotted in the paper. The results are compared with that obtained from the original structure. As shown, when segmented or compact arrangement is adopted, the suppressing of vibrations is not obvious because the damping ratios only increase slightly in these cases as shown in Fig. 12(b). Moreover, it can be seen that more constraining segments do not necessarily result in more effective vibration reduction. This is most evident for the case where the pipeline is compactly assembled with five segments $(\mathrm{L}=10 \mathrm{~m})$. This system vibrates even more violently than the original pipeline. This is because the addition of the segments obviously changes the vibration frequency of the system while it does not increase the damping evidently. As can be 
seen from Fig. 12(a), the vibration frequencies for the original pipeline and the pipeline with five compact segments are 3.8556 and $3.0120 \mathrm{~Hz}$ respectively. Moreover, Fig. 8 shows that the energy of the earthquake loading mainly concentrates around $2.734 \mathrm{~Hz}$ due to the local site amplification effect. This frequency is close to the vibration frequency of the compact scenario (3.0120 Hz), which means that when the pipeline is compactly assembled with five segments, resonance can occur and larger seismic response is expected. When monolithic arrangement is adopted, the reduction of vibration is significant as shown in Fig. 13(c) due to the obvious increment of damping (see Fig. 12(b)). Of course, the decreased vibration frequency makes the system vibrates closer to the resonant frequency of local soil site, however, this effect is compensated by the increased damping ratio of the system. The results suggest that monolithic arrangement is more effective than the segmented and compact arrangements in the seismic vibration control of above-ground pipelines.

\subsection{Influence of constraining length}

To investigate the influence of constraining length on the modal parameters and seismic responses, eight different cases are investigated. The constraining length ranges from 2 to 16 $\mathrm{m}$ (the whole span is constrained) with an increment of $2 \mathrm{~m}$. Only the monolithic arrangement is considered from this section since it is more effective compared to the segmented and compact arrangements as discussed above. The results with the constraining lengths of 0 (original pipeline), 2, 6 and $10 \mathrm{~m}$ have been presented in Section 4.1, they are presented again in this section for comparison purpose. The thickness of the VEMs is $20 \mathrm{~mm}$ and the thickness of the CLs is $3 \mathrm{~mm}$. The constraining angle is $72^{\circ}$.

Fig. 14(a) shows the influence of constraining length on the vibration frequency of the system. As shown, the vibration frequency decreases with the increment of constraining length if the constraining length is less than $6 \mathrm{~m}$ due to its evident contribution on the mass of the system. When the constraining length is larger than $6 \mathrm{~m}$, the constraining layers contribute more to the stiffness compared to their contributions to the mass, the vibration frequency increases with the increment of constraining length as discussed above. For the damping ratio of the system, Fig. 14(b) shows that when the constraining length is less than $10 \mathrm{~m}$, the damping ratio increases with the increment of constraining length. When the constraining length is larger than $10 \mathrm{~m}$, the damping ratio, however, decreases with the increment of constraining length. For example, when the constraining length is $10 \mathrm{~m}$, the damping ratio is $4.78 \%$. When the pipeline is fully constrained $(\mathrm{L}=16 \mathrm{~m})$, the damping ratio 
reduces to $3.83 \%$. This observation coincides with the analytical results obtained by Wang and Chen [22], where they found that the influence of constraining length on the damping ratio of the system is related to the boundary conditions of the system. They found that when the structure is simply-supported, increasing the constraining length will correspondingly increase the damping ratio. However, when the structure is partially or fully clamped, longer constraining layer does not necessarily introduce larger damping to the system. In the present study, two rotational springs are added at the end of the single-span model to account for the influence of adjacent spans. Since the structure is partially restrained, similar results obtained by Wang and Chen [22] are thus observed.

Fig. 15 compares the displacement time histories at the middle span of the original pipeline and the pipelines assembled with different lengths of constraining layers. It can be seen that when the constraining length is larger than $6 \mathrm{~m}$, the vibration reduction is obvious due to the significant increment of damping in the system as shown in Fig. 14(b). When the length of the constraining layers reaches a certain level, increasing its length will not significantly increase, and in some cases may even slightly decrease its effectiveness on vibration mitigation. This can be explained by the combined influences of the vibration frequency and damping ratio. The results show that properly selected constraining layers will not only maximize its effectiveness on the vibration mitigation but also can save the materials and thus decrease the cost.

\subsection{Influence of constraining angle}

The influence of constraining angle $\alpha$ on the modal parameters and seismic responses of the system is investigated in this section. Six different constraining angles, ranging from $0^{\circ}$ (original structure) to $90^{\circ}$ with an interval of $18^{\circ}$, are investigated. For each case, the constraining length is $8 \mathrm{~m}$, the thickness of the VEMs is $20 \mathrm{~mm}$ and the thickness of the CLs is $3 \mathrm{~mm}$.

Fig. 16(a) shows the influence of constraining angle on the vibration frequency of the system. It can be seen that increasing the constraining angle almost linearly decreases the vibration frequency of the system owing to the obvious increment of mass to the system. On the other hand, increasing the constraining angle does not increase the damping ratio linearly but seems parabolically. When the angle is small, the influence of constraining angle on the damping ratio is obvious. When the constraining angle is relatively large, the increment becomes smaller. This is because the damping in the transverse direction is investigated in 
the present paper, the shear deformations of the VEMs close to the horizontal plane contribute more to the damping ratio. The contributions from the parts which are far from the horizontal plane are unobvious. As for the displacement response, it seems that the constraining angle of $54^{\circ}$ is an optimal value to suppress the transverse vibration of the system in terms of the material costs and vibration supress effectiveness, as shown in Fig. 17.

\subsection{Influence of VEM thickness}

To study the influence of VEM thickness on the modal parameters and seismic response, five different cases are studied. The VEM thickness varies from 0 (i.e., the CLs are directly connected to the original pipeline) to $40 \mathrm{~mm}$ with an interval of $10 \mathrm{~mm}$. The constraining length is $8 \mathrm{~m}$, the thickness of the CLs is $3 \mathrm{~mm}$ and the constraining angle is $72^{\circ}$. For comparison, the corresponding values for the original pipeline are also shown and they are marked as star in Fig. 18.

Fig. 18(a) shows the influence of VEM thickness on the vibration frequency of the system. As shown, direct addition of CLs to the original pipeline leads to the highest vibration frequency of the system. The vibration frequency decreases obviously when a layer of VEM is attached to the system. When the CLs are directly connected to the original pipeline, the vibration frequency is $3.9946 \mathrm{~Hz}$, while it reduces to $3.4572 \mathrm{~Hz}$ when a $10 \mathrm{~mm}$ VEM layer is attached between the original pipeline and the CLs. This is because the Young's modulus of the VEMs (6.88MPa) is much smaller than that of the steel CLs (210 $\mathrm{GPa}$ ). The addition of the VEMs does not prominently add the stiffness but increases the mass of the system. Further increasing the thickness of the VEMs (almost linearly) reduces the vibration frequency of the system.

For the damping ratio of the system, Fig. 18(b) shows that when the CLs are directly attached to the original pipeline, the identified damping ratio is $1.25 \%$, which is the same as the original pipeline system since both the original pipeline and the CLs are made of the same steel. The damping ratio increases sharply to $4.05 \%$ when a $10 \mathrm{~mm}$ VEM is assembled to the system, which means the addition of even a thin layer of VEMs can obviously increase the damping ratio of the system. Further increasing the VEM thickness (almost linearly) increases the damping ratio of the system. The increasing rate is, however, reduced. For example, the damping ratio is $5.07 \%$ when the VEM thickness is $40 \mathrm{~mm}$. The increment of VEM thickness by $30 \mathrm{~mm}$ (from 10 to $40 \mathrm{~mm}$ ) only increases the damping ratio by $1.02 \%$ (from $4.05 \%$ to $5.07 \%$ ). 
Fig. 19 shows the influence of VEM thickness on the seismic response of the system. It can be seen that when the CLs are directly attached to the original pipeline, the vibration of the original pipeline is also reduced especially during the time interval from 8 to 12 sec though the damping ratio of the system is the same as the original pipeline. This can be explained by the energy of the earthquake loading and the vibration frequency of the system. As mentioned above, the vibration frequencies of the original pipeline and that with directly assembled CLs are 3.8556 and $3.9946 \mathrm{~Hz}$ respectively. The energy of the earthquake loading at $3.9946 \mathrm{~Hz}$ is slightly smaller than that at $3.8556 \mathrm{~Hz}$ as shown in Fig. 8, which in turn also leads to the smaller seismic response. The figure also shows that the addition of VEMs can effectively reduce the vibration of the system due to the obvious increment of the damping to the system. However, it does not mean the thicker is the VEM, the better is its effectiveness on the vibration control. Actually it seems that the vibration of the system assembled with 40 $\mathrm{mm}$ VEMs is larger than that assembled with $20 \mathrm{~mm}$ and $30 \mathrm{~mm}$ VEMs though the damping ratio is larger compared to those two. This can be explained by the resonance effect again.

\subsection{Influence of CL thickness}

The influence of CL thickness on the modal parameters and seismic responses is investigated in this section. Six different cases are considered. The CL thickness varies from 0 (only the VEM is assembled to the system) to $5 \mathrm{~mm}$ with an interval of $1 \mathrm{~mm}$. The thickness of the VEM is $20 \mathrm{~mm}$, the constraining length is $8 \mathrm{~m}$ and the constraining angle is $72^{\circ}$ in these cases. Fig. 20 shows the variations of the vibration frequency and damping ratio of the pipeline model with respect to the CL thickness. For comparison, the corresponding values for the original pipeline are also shown and marked as star in Fig. 20.

Fig. 20(a) shows the influence of CL thickness on the vibration frequency of the system. As shown, the vibration frequency of the system with VEM assembled only is smaller than that of the original pipeline due to the added mass from the VEM and relatively small contribution to the stiffness. When $1 \mathrm{~mm}$ CLs are assembled, the vibration frequency increases owing to the stiffness contribution from the added CLs. The vibration frequency then decreases with the increasing of the CL thickness. For the damping ratio as shown in Fig. 20(b), it is interesting to find that if the CLs are not added to the system, the damping ratio is the same as the original pipeline though the high damping VEMs are added. The damping ratio of the system increases evidently with the increase of CL thickness. As shown, when the CL thickness is $1 \mathrm{~mm}$, the damping ratio of the system is $2.40 \%$ and it reaches $5.48 \%$ if the 
CL thickness increases to $5 \mathrm{~mm}$. It reiterates from Section 4.4 that the increment of $30 \mathrm{~mm}$ VEM thickness only leads to a $1.02 \%$ increasing of damping ratio to the system. Increasing CL thickness is more effective to increase the damping ratio of the system than increasing the VEM thickness. This might be because CL is much stiffer than VEM, a slight increasing in the CL thickness can provide much stronger constraint to the VEM, which in turn significantly increases the shear deformation of the VEM and thus leads to larger damping ratio. On the other hand, when the thickness of the CL is fixed, the constraint provided by the CL is fixed, the increment on the VEM shear deformation is not evident thought the VEM thickness is increased significantly.

Fig. 21 shows the influence of CL thickness on the seismic response of the system. When only the VEM is attached to the original pipeline, the vibration of system is more severe than the original pipeline. This is because the damping ratio is not increased as mentioned above while the vibration frequency of the system is closer to the resonant frequency of local site. The addition of the CL can obviously suppress the vibration of the system due to the increased damping ratio. However, when the thickness of CL is larger than $3 \mathrm{~mm}$, it seems that further increasing the CL thickness is not very effective to suppress the vibration of the system though the damping is increased. This is due to the change of the vibration frequency and thus the vibration characteristic of the system.

\subsection{Influence of different earthquake loadings}

In the previous sections, the artificially simulated earthquake loading is used as input in the numerical simulation. To further examine the influence of ground motion frequency content on the effectiveness of the proposed method, the seismic responses of the original and constrained pipelines subjected to two natural earthquake loadings obtained from the database of Pacific Earthquake Engineering Research Centre (PEER [33]) are also calculated and compared. The first earthquake loading is the record from the 1994 Northridge earthquake. This earthquake loading is characterized by the long-period pulse-like waveforms, and it is normally classified as near-fault ground motion. The second record is from the 1971 San Fernando earthquake, which exhibits fewer long-period characteristics and it is used to represent far-field earthquake. Table 5 summarises these two earthquake components and Fig. 22 shows the accelerograms of the two ground motions. Fig. 23 plots the PSDs of these two earthquake loadings. It can be seen that the energies of the Northridge earthquake loading mainly concentrate in the frequency band less than $2 \mathrm{~Hz}$ and for the San Fernando earthquake, 
they are mainly in the frequency band less than $1 \mathrm{~Hz}$. The dominant frequencies of these two earthquakes are far from the first vibration frequencies of the original and constrained pipelines, which are 3.8556 and $3.3747 \mathrm{~Hz}$ respectively as mentioned above. The changes in the seismic responses are thus mainly because of the change of the damping. Fig. 24 shows the seismic responses of the original and constrained pipelines subjected to these two earthquake loadings. It is obvious that the proposed method is effective to suppress the vibrations induced by these two natural earthquake loadings. It is noted that the corresponding parameters for the constraining layers are: VEM thickness $=20 \mathrm{~mm}$, CL thickness $=3 \mathrm{~mm}$, constraining length $=8 \mathrm{~m}$ and constraining angle $=72^{\circ}$.

The presented numerical results show that with properly selected VEMs and CLs, the proposed method can be used to effectively mitigate the seismic induced vibrations of aboveground pipelines. Its effectiveness on the vibration control depends on the increased damping ratio and the changed vibration characteristic of the system. For the considered example, the VEMs and CLs with length of $8 \mathrm{~m}$, constraining angle of 54 $4^{\circ}$, VEM thickness of $20 \mathrm{~mm}$ and CL thickness of $3 \mathrm{~mm}$ is found to be a good choice in terms of vibration control and cost for the considered pipeline system. The proposed method has following obvious features: (1) with this design, the mass of one span pipeline increases from 408 to $512 \mathrm{~kg}$, with an increment of 25\%; the vibration frequency reduces from 3.8556 to $3.4598 \mathrm{~Hz}$ with a decrement of $10 \%$; the damping ratio, however, increases from $1.25 \%$ to $3.96 \%$ with an significant increment of $217 \%$; (2) with a $25 \%$ mass increment and $10 \%$ vibration frequency reduction, the original design of the pipeline is believed not necessary to be revised; (3) VEMs are cheap method to increase the damping of engineering structures [15, 34], the cost on the VEMs will be limited; (4) the VEMs and CLs can be directly assembled to the original system, this method can be conveniently implemented with limited man power cost. These features indicate that the effectiveness of this vibration control solution will be obvious, whereas the cost will be relatively low, especially one considers the enormous economy loss and detrimental environmental impact that may be caused by the damage of a lifeline pipeline system during a severe earthquake.

In engineering practice, the influence of temperature and the durability of VEMs may be of great concern. In the present study, VHB4955 manufactured by $3 \mathrm{M}{ }^{\circledR}$ is used as VEMs. $3 \mathrm{M}{ }^{\circledR}$ indicates that this material is effective within the temperature range from -40 to $93^{\circ} \mathrm{C}$ (http://www.uline.com/Product/Detail/S-10148/3M-VHB-Tapes/3M-4955-VHB-Double- 
Sided-Foam-Tape-1-2-x-36-yds.), which means this material can be applied in most of the extreme temperature conditions in engineering practice. $3 \mathrm{M} \AA$ also published a technical bulletin to demonstrate the long-term durability of VHB tapes to various harsh environmental conditions (http://multimedia.3m.com/mws/media/98989O/vhbtm-durability.PDF). The test results suggest very encouraging durability performance with many of them survived more than 20 years. Based on the discussion above, it is believed that VHB4955 manufactured by $3 \mathrm{M}{ }^{\circledR}$ can be used as the VEMs. However, it should be noted that this material is selected in the present study is simply because Borges et al. [16] used this material and the properties of this material are well defined. Other VEMs can also be the options in engineering practices.

\section{Conclusions}

This paper carries out numerical simulations on the effectiveness of using viscoelastic materials to mitigate seismic induced vibrations of above-ground pipeline structures. The numerical analyses are carried out by using the commercial software package ANSYS. The modelling of the viscoelastic material is firstly calibrated based on the experimental data obtained from testing a $1.6 \mathrm{~m}$ long tubular sandwich structure. The calibrated material model is then applied to the above-ground pipeline system. Various parameters including the constraining arrangements, the constraining length and angle, the thicknesses of the VEMs and CLs are investigated in detail. The influence of earthquake frequency content is also examined. Following conclusions are obtained:

1. It is effective to mitigate the seismic induced vibrations of above-ground pipelines by directly assembling the VEMs and CLs to the original pipeline structure. Its effectiveness on the vibration control depends on the increased damping ratio and the changed vibration characteristic of the system.

2. The monolithic arrangement is more effective than segmented and compact arrangements in increasing the damping of the original pipeline system and suppressing its vibration.

3. Increasing the length of the constraining layers does not necessarily increase the damping ratio of the pipeline system monotonously due to the influence of adjacent spans.

4. Increasing the constraining angle will increase the damping of the system. When the angle is small, increasing the constraining angle is effective in increasing the damping ratio. When the constraining angle is relatively large, further increasing the angle is less effective in increasing the damping. 
5. The damping of the original pipeline can be increased significantly by adding even a thin layer of VEMs to the system. Further increasing VEM thickness from $10 \mathrm{~mm}$ always increases the damping ratio of the system but is less effective.

6. When a layer of VEM is assembled to the original pipeline, increasing CL thickness can evidently increase the damping ratio of the system. However, when the thickness of CL reaches certain level, further increasing the CL thickness is no longer very effective in suppressing the vibration of the pipeline system.

\section{Acknowledgement}

The first author would like to acknowledge the support from Australian Research Council Discovery Early Career Researcher Award DE150100195 for carrying out this research.

\section{References}

[1]Makar JM, Desnoyers R, McDonald SE. Failure modes and mechanisms in gray cast iron pipe. In: Proceedings of international conference on underground infrastructure research, Waterloo, Canada: 2001. p. 1-10.

[2]O’Rourke MJ, Liu X. Response of buried pipelines subjected to earthquake effects. New York: Multidisciplinary Centre for Earthquake Engineering Research; 1999.

[3] Lanzano G, Salzano E, Santucci de Magistris F, Fabbrocino G. Seismic vulnerability of gas and liquid buried pipelines. J. Loss Prev. Process Indust 2014; 28: 72-8.

[4]Arjomandi K, Taheri F. Bending capacity of sandwich pipes. Ocean Eng 2012; 48: 17-31.

[5]Khalilpasha H, Albermani F. Textured deep subsea pipelines. Int J Mech Sci 2013; 68: 224-35.

[6]Kumar RA, Sohn CH, Gowda BHL. Passive control of vortex-induced vibrations: an overview. Recent Pat Mech Eng 2008; 1: 1-11.

[7]Bi K, Hao H. Using pipe-in-pipe systems for subsea pipeline vibration control. Eng Struct 2016; 109: 75-84.

[8]Norris MA, Ptak KR, Zamora BA, Hart JD. Implementation of tuned vibration absorbers for above ground pipeline vibration control. In: Proceedings of the international pipeline conference, Calgary, Canada: 2000. IPC00-0270.

[9]Powell GH. Seismic response analysis of above-ground pipelines. Earthquake Eng Struct Dyn 1978; 6: 157-65. 
[10] Anderson JC, Johnston SB. Seismic behaviour of above-ground oil pipeline. Earthquake Eng Struct Dyn 1975; 3: 319-36.

[11] Soliman HO, Datta TK. Response of overground pipelines to random ground motion. Eng Struct 1996; 18(7): 537-45.

[12] Lanzano G, Santucci de Magistris F, Fabbrocino G, Salzano E. Seismic damage to pipelines in the framework of Na-Tech risk assessment. J. Loss Prev. Process Indust 2015; 33: 159-72.

[13] Soong TT, Spencer BF. Supplemental energy dissipation: state-of-the-art and stateof-the-practice. Eng Struct 2002; 24: 246-59.

[14] Norris MA, Williams SL, Ptak KR, Mazzoleni AP, Hahn GD, Masson C. Passive vibration control treatment for subsea pipeline. In: Proceedings of offshore technology conference, Rio de Janeiro, Brazil: 2013. Paper No. OTC24314.

[15] Saidi I, Gad EF, Wilson JL, Haritos N. Development of passive viscoelastic damper to attenuate excessive floor vibrations. Eng Struct 2011; 33: 3317-28.

[16] Borges FCL, Riotman N, Magluta C, Castello DA, Franciss R. A concept to reduce vibrations in steel catenary risers by the use of viscoelastic materials. Ocean Eng 2014; 77: $1-11$.

[17] Samali B, Kwok KCS. Use of viscoelastic dampers in reducing wind- and earthquakeinduced motion of building structures. Eng Struct 1995; 17: 639-54.

[18] Min KW, Kim J, Lee SH. Vibration tests of 5-story steel frame with viscoelastic dampers. Eng Struct 2004; 26: 831-9.

[19] Kerwin EM. Damping of flexural waves by a constrained viscoelastic layer. J Acoust Soc Am 1959; 31: 952-62.

[20] Ross D, Ungar EE, Kerwin EM. Damping of plate flexural vibrations by means of viscoelastic laminate. Struct Damping Sect ASME 1959; 3: 49-88.

[21] Chen LH, Huang SC. Vibrations of a cylindrical shell with partially constrained layer damping (CLD) treatment. Int J Mech Sci 1999; 41: 1485-98.

[22] Wang HJ, Chen LW. Finite element dynamic analysis of orthotropic cylindrical shells with a constrained damping layer. Finite Elem Anal Des 2004; 40: 737-55.

[23] ANSYS. ANSYS user manual. ANSYS, Inc; 2002.

[24] Menard KP. Dynamic mechanical analysis a practical introduction. Boca Raton, Fla: CRC Press; 1999. 
[25] Stutz LT, Magluta C, Roitman N, Silva RP. Experimental and numerical analysis of a sandwich beam with viscoelastic layer. In: Proceedings of the $20^{\text {th }}$ international congress of mechanical engineering, Gramado, Brazil: 2009.

[26] Nashif AD. Vibration damping. New York: John Wiley \& Sons; 1985.

[27] Ruzzene M, Fasana A, Garibaldi L, Piombo B. Natural frequencies and dampings identification using wavelet transform: application to real data. Mech Syst Signal Pr 1997; 11(2): 207-18.

[28] Tajimi H. A statistical method of determining the maximum response of a building structure during an earthquake. In: Proceedings of 2nd World Conference on Earthquake Engineering, Tokyo, 1960; 781-96.

[29] Der Kiureghian A. Structural response to stationary excitation. J Eng Mech Div 1980; 106(6): 1195-213.

[30] $\mathrm{Bi} \mathrm{K}$, Hao H. Modelling and simulation of spatially varying earthquake ground motions at sites with varying conditions. Probab Eng Mech 2012; 29: 92-104.

[31] Wolf JP. Dynamic soil-structure interaction. New Jersey: Englewood Cliffs; 1985.

[32] Bao C, Hao H, Li ZX. Integrated ARMA model method for damage detection of subsea pipeline system. Eng Struct 2013; 48: 176-92.

[33] Pacific Earthquake Engineering Research Centre (PEER) Ground Motion Database. http://ngawest2.berkeley.edu/.

[34] Ljunggren F, Agren A. Development of a new damper to reduce resonant vibrations in lightweight steel joist floors. Appl Acoust 2002; 63: 1267-80. 
Table 1. Geometric properties of the tube layers [16]

\begin{tabular}{|c|c|c|c|}
\hline Layer (material) & Length (mm) & External radius (mm) & Thickness (mm) \\
\hline Internal tube (Brass) & 1600 & 9.46 & 1.06 \\
\hline Viscoelastic material (VHB4955) & 1600 & 11.86 & 2.4 \\
\hline Constraining (Brass) & 1600 & 12.66 & 0.8 \\
\hline
\end{tabular}

Table 2. Comparison of the modal parameters identified from the numerical results and the corresponding experimental results (the original tube)

\begin{tabular}{|c|c|c|c|c|c|c|c|c|}
\hline \multirow[b]{2}{*}{$\begin{array}{l}\text { Mode } \\
\text { No }\end{array}$} & \multicolumn{2}{|c|}{ Numerical results } & \multicolumn{2}{|c|}{ Experimental results [16] } & \multicolumn{4}{|c|}{ Difference (\%) } \\
\hline & $\begin{array}{c}\text { Frequency } \\
(\mathrm{Hz})\end{array}$ & $\begin{array}{c}\text { Damping ratio } \\
(\%)\end{array}$ & $\begin{array}{c}\text { Frequency } \\
(\mathrm{Hz})\end{array}$ & $\begin{array}{c}\text { Damping ratio } \\
(\%)\end{array}$ & Frec & ency & Dam & ratio \\
\hline 1 & & & & & -0.39 & 0.59 & 40 & 40 \\
\hline 2 & & & & & -0.90 & -0.22 & 50 & 100 \\
\hline
\end{tabular}

Table 3. Comparison of the modal parameters identified from the numerical results and the corresponding experimental results (the tube with faces 1 and 2 constrained)

\begin{tabular}{|c|c|c|c|c|c|c|c|c|}
\hline \multirow[b]{2}{*}{$\begin{array}{l}\text { Mode } \\
\text { No }\end{array}$} & \multicolumn{2}{|c|}{ Numerical results } & \multicolumn{2}{|c|}{ Experimental results [16] } & \multicolumn{4}{|c|}{ Difference (\%) } \\
\hline & $\begin{array}{c}\text { Frequency } \\
(\mathrm{Hz})\end{array}$ & $\begin{array}{c}\text { Damping ratio } \\
(\%)\end{array}$ & $\begin{array}{c}\text { Frequency } \\
(\mathrm{Hz})\end{array}$ & $\begin{array}{c}\text { Damping ratio } \\
\text { (\%) }\end{array}$ & & ency & Dampi & rati \\
\hline 1 & & & & & 5.36 & 6.62 & -10.02 & -4.00 \\
\hline 2 & & & & & 7.08 & 7.20 & -8.88 & 24.24 \\
\hline
\end{tabular}

Table 4. Comparison of the modal parameters identified from the numerical results and the corresponding experimental results (the tube with all faces constrained)

\begin{tabular}{|c|c|c|c|c|c|c|c|c|}
\hline \multirow[b]{2}{*}{$\begin{array}{l}\text { Mode } \\
\text { No }\end{array}$} & \multicolumn{2}{|c|}{ Numerical results } & \multicolumn{2}{|c|}{ Experimental results [16] } & \multicolumn{4}{|c|}{ Difference (\%) } \\
\hline & $\begin{array}{c}\text { Frequency } \\
(\mathrm{Hz})\end{array}$ & $\begin{array}{c}\text { Damping ratio } \\
(\%)\end{array}$ & $\begin{array}{c}\text { Frequency } \\
(\mathrm{Hz})\end{array}$ & $\begin{array}{c}\text { Damping ratio } \\
(\%)\end{array}$ & Fre & ency & Damp & ratio \\
\hline 1 & & & & & 10.19 & 12.84 & -14.40 & 9.04 \\
\hline 2 & & & & & 11.26 & -18.04 & -13.74 & 1.49 \\
\hline
\end{tabular}


Table 5. Two natural earthquake records

\begin{tabular}{|c|c|c|c|}
\hline Earthquake & Date & Station & Component \\
\hline Northridge & $17 / 01 / 1994$ & Sylmar & NS \\
\hline San Fernando & $09 / 02 / 1971$ & 2516 Via Tejon PV & NS \\
\hline
\end{tabular}




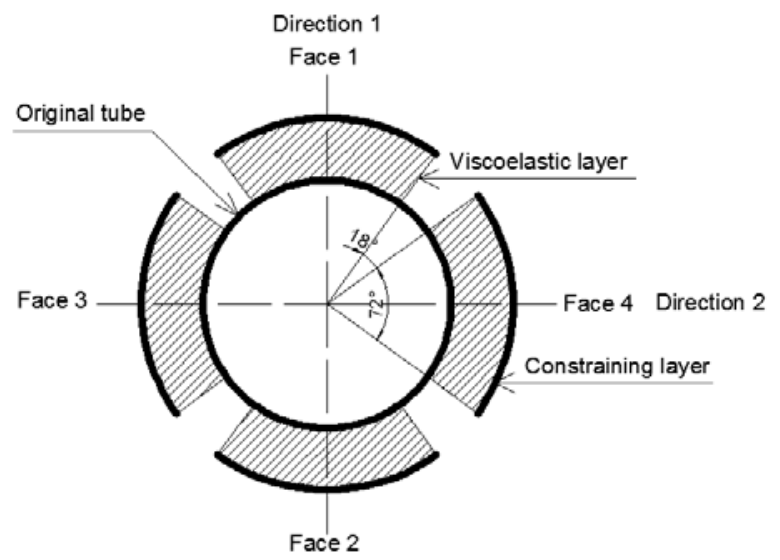

Fig. 1. Tubular cross section of the sandwich beam structure (not to scale, after [16])

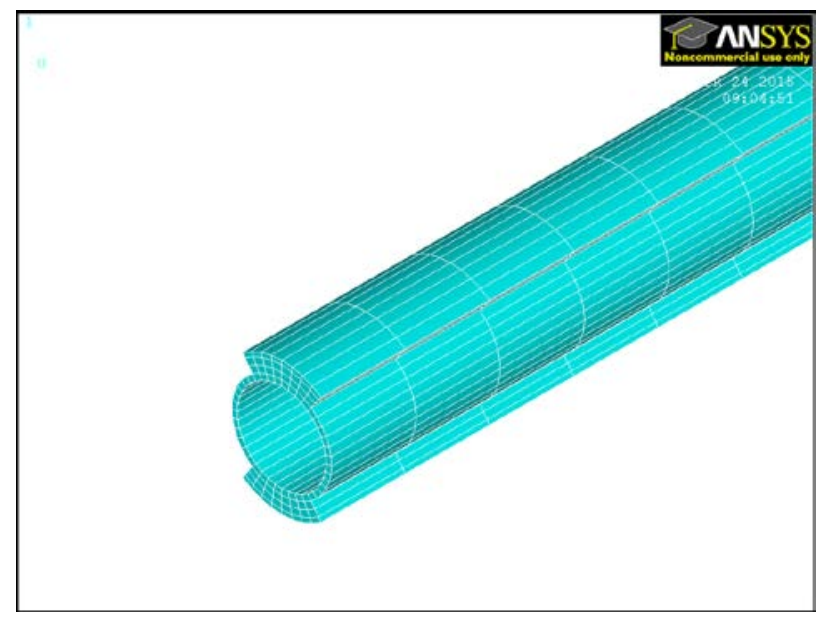

Fig. 2. FE model of the original tube with faces 1 and 2 constrained with VEMs and CLs

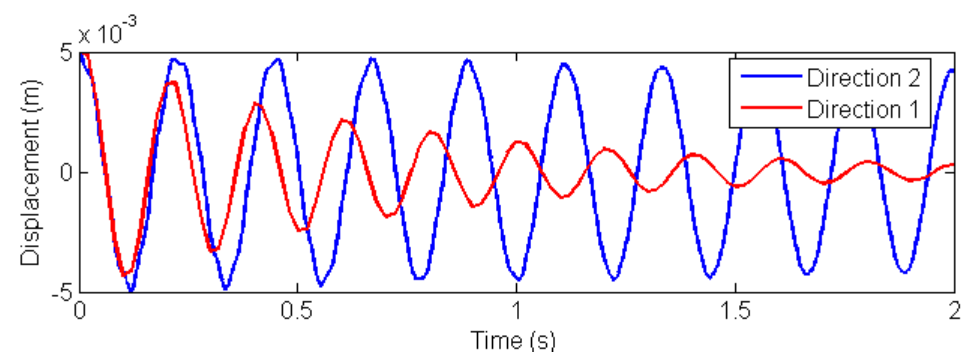

Fig. 3. Free vibration displacement time histories at different directions of the constrained sandwich system 


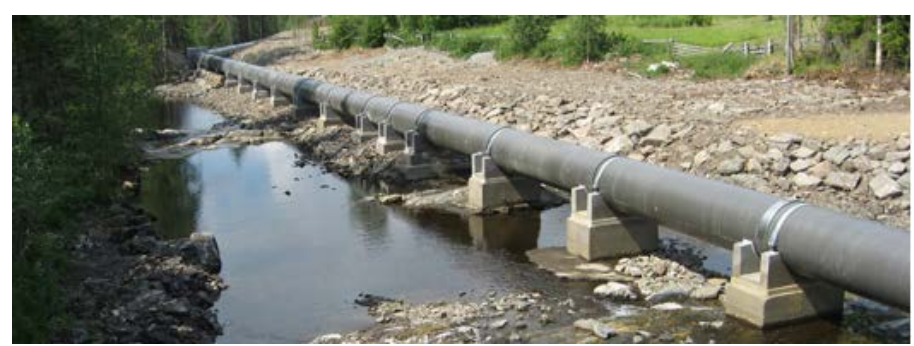

Fig. 4. A typical above-ground pipeline

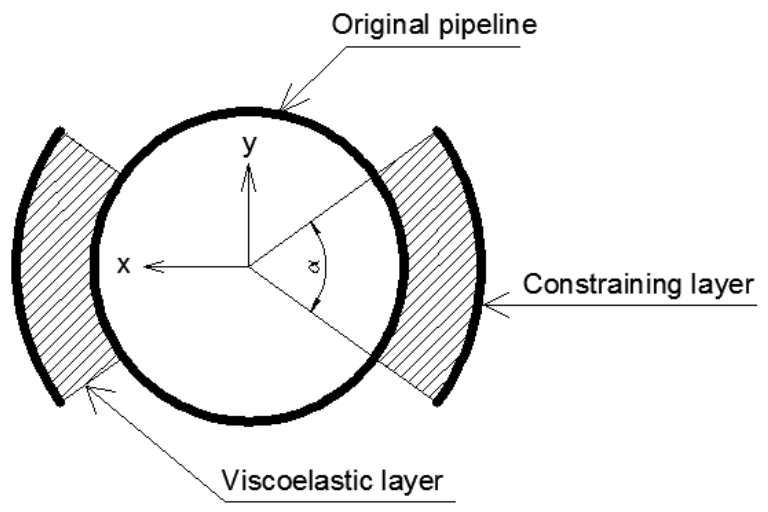

Fig. 5. Cross section of the constrained pipeline

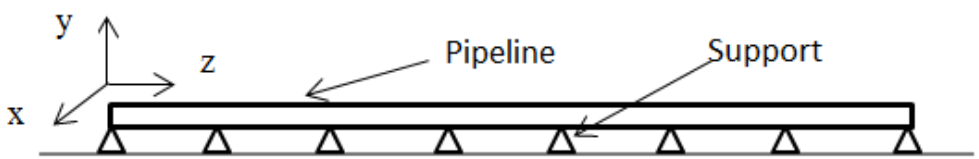

Soil layer: $\rho_{s}=2000 \mathrm{~kg} / \mathrm{m}^{3}, G_{s}=200 \mathrm{MPa}, \zeta_{s}=5 \%, v_{s}=0.4, h=30 \mathrm{~m}$

Base rock: $\rho_{b}=3000 \mathrm{~kg} / \mathrm{m}^{3}, G_{b}=1800 \mathrm{MPa}, \zeta_{b}=5 \%, v_{b}=0.33$

Fig. 6. Above-ground pipeline and underneath site conditions (not to scale) 


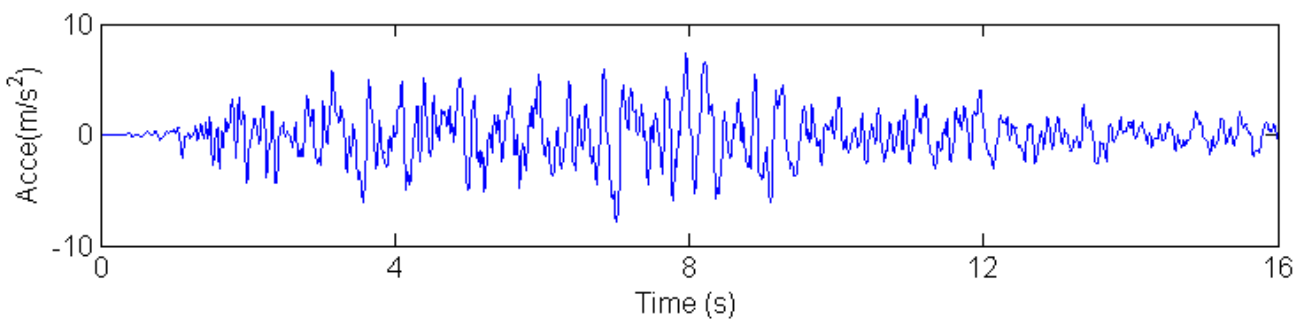

Fig. 7. Simulated transverse acceleration time history

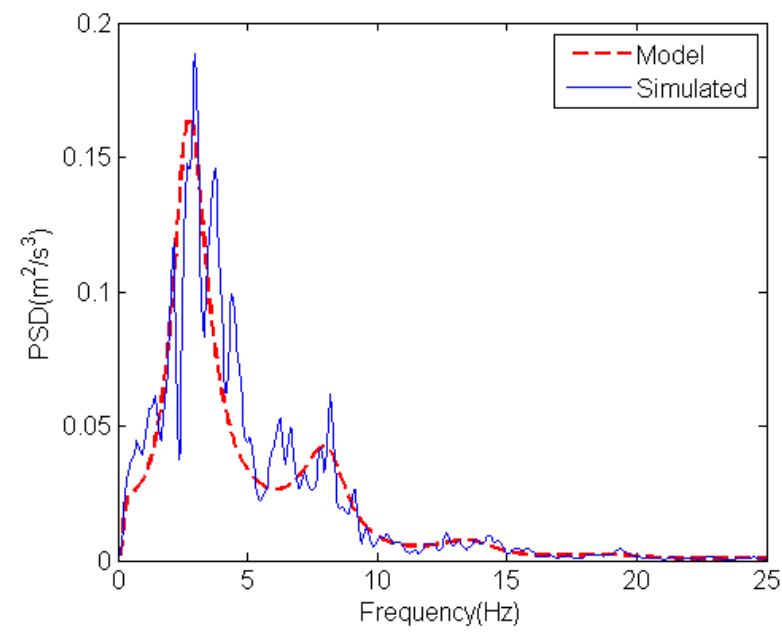

Fig. 8. Comparison of the PSDs of the simulated ground motion with the theoretical model

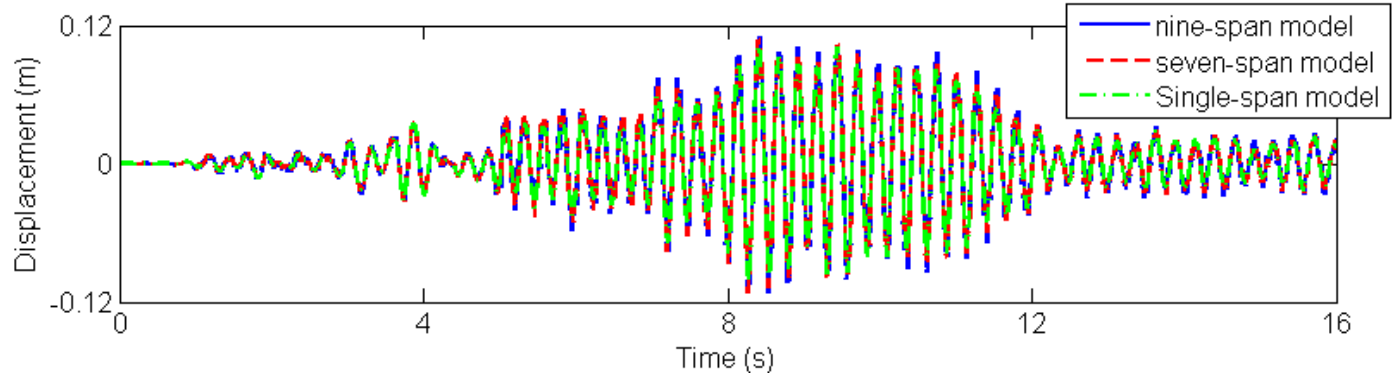

Fig. 9. Displacement responses of the middle point obtained from different models 


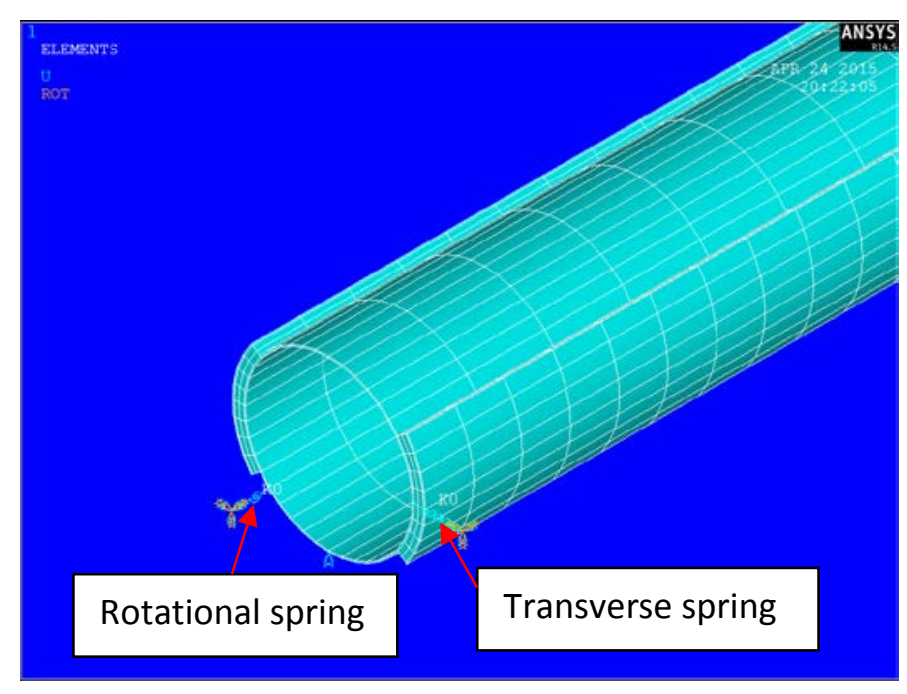

Fig. 10. Finite element model of a single-span pipeline with the whole span assembled with VEMs and CLs

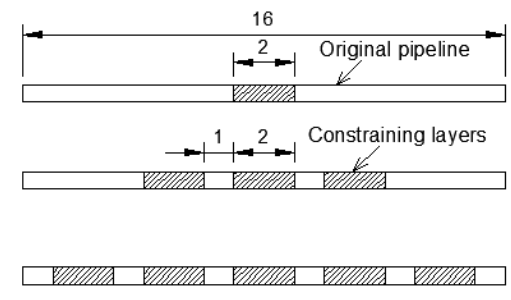

(a) Segmented arrangement

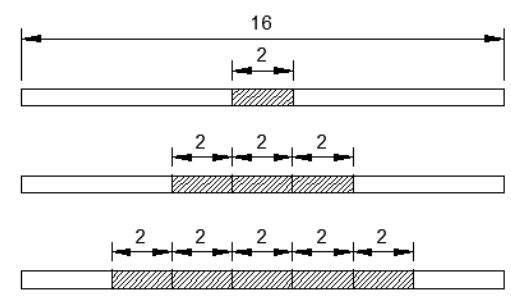

(b) Compact arrangement

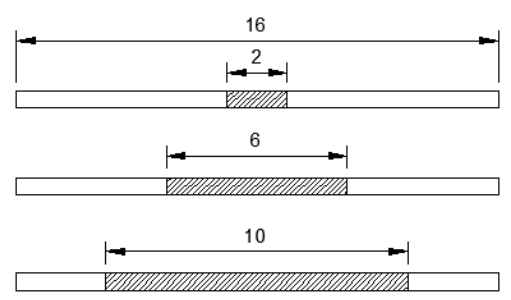

(c) Monolithic arrangement

Fig. 11. Constraining arrangement scenarios: (a) segmented, (b) compact and (c) monolithic configurations (unit: meter) 
(a)

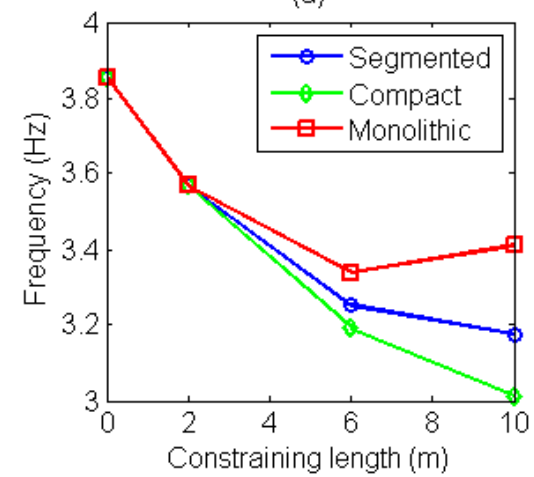

(b)

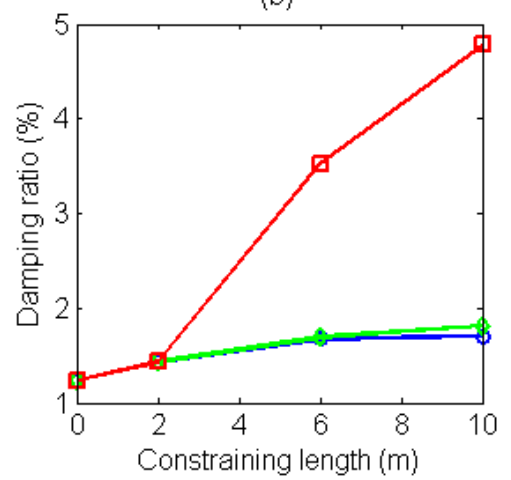

Fig. 12. Influence of constraining arrangement scenarios on the modal parameters of the system: (a) vibration frequency and (b) damping ratio

(a) Segmented
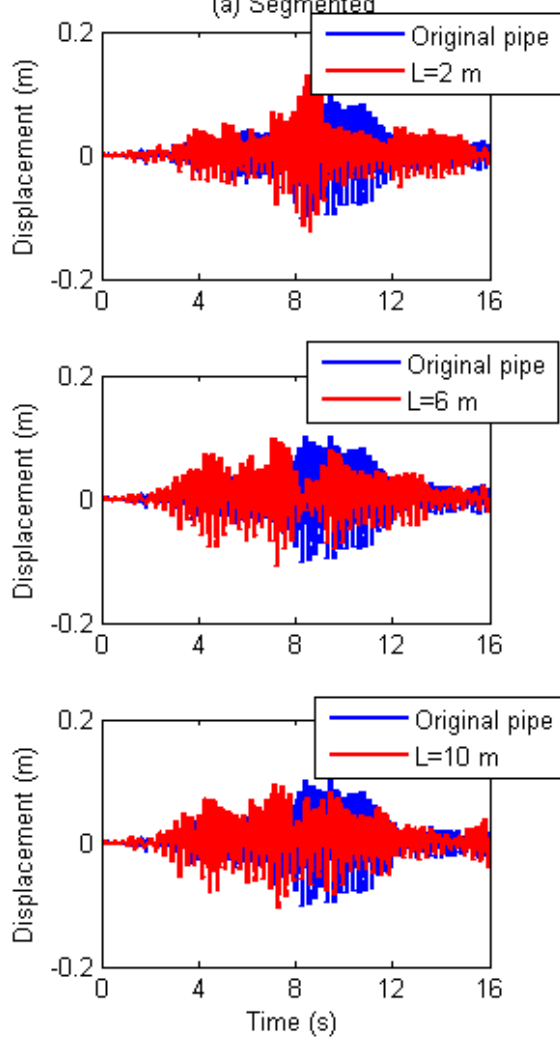

(b) Compact
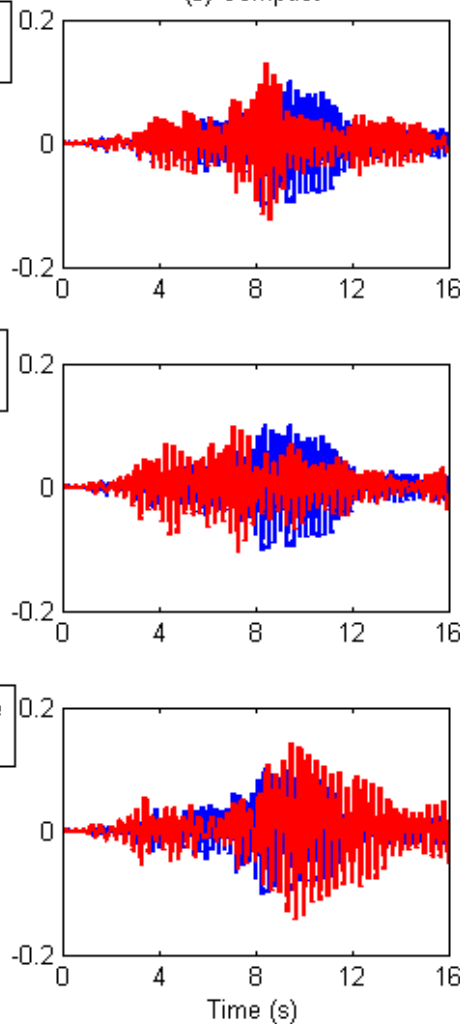

(c) Monolithic
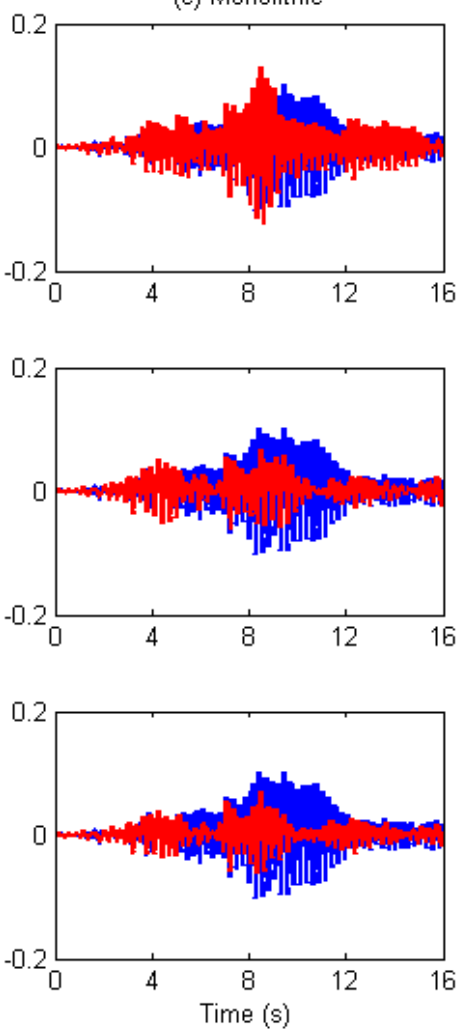

Fig. 13. Influence of constraining arrangement scenarios on the seismic responses of the system: (a) segmented, (b) compact and (c) monolithic configurations 
(a)

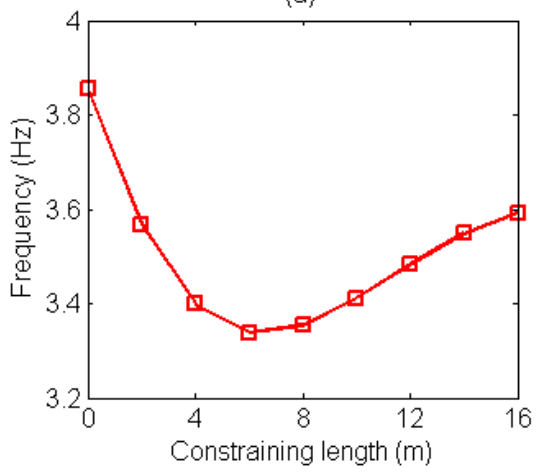

(b)

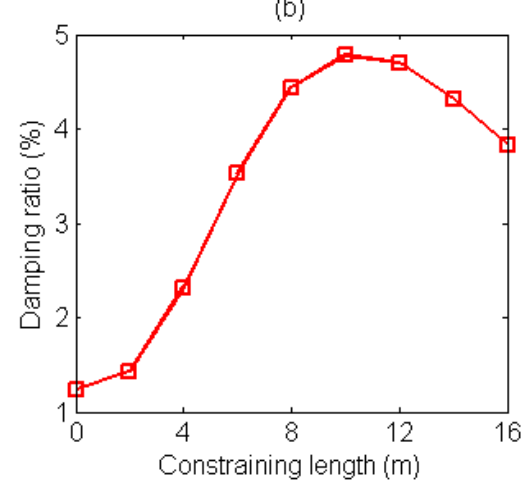

Fig. 14. Influence of constraining length on the modal parameters of the system: (a) vibration frequency and (b) damping ratio
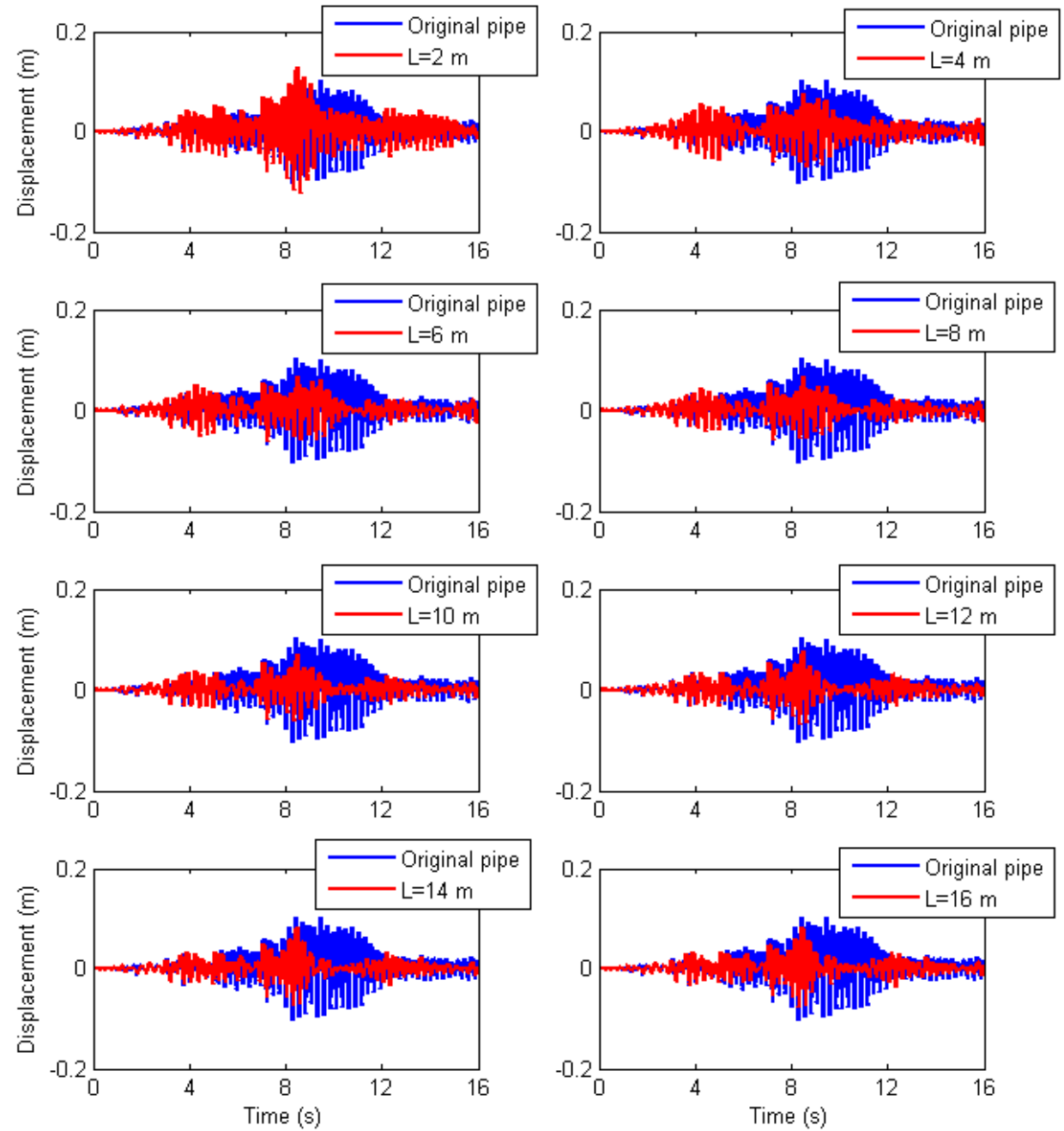

Fig. 15. Influence of constraining length on the seismic responses of the system 
(a)

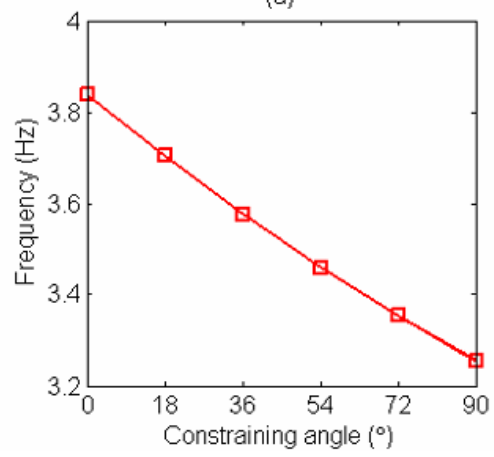

(b)

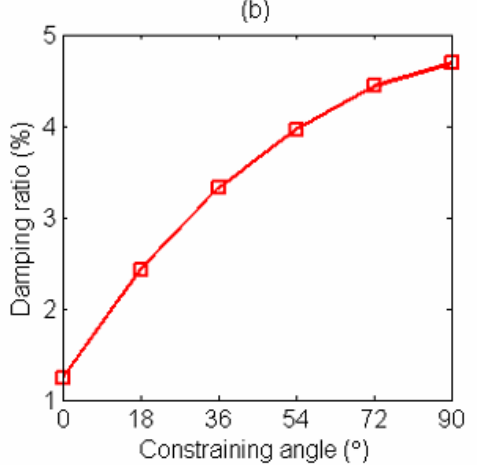

Fig. 16. Influence of constraining angle on the modal parameters of the system: (a) vibration frequency and (b) damping ratio
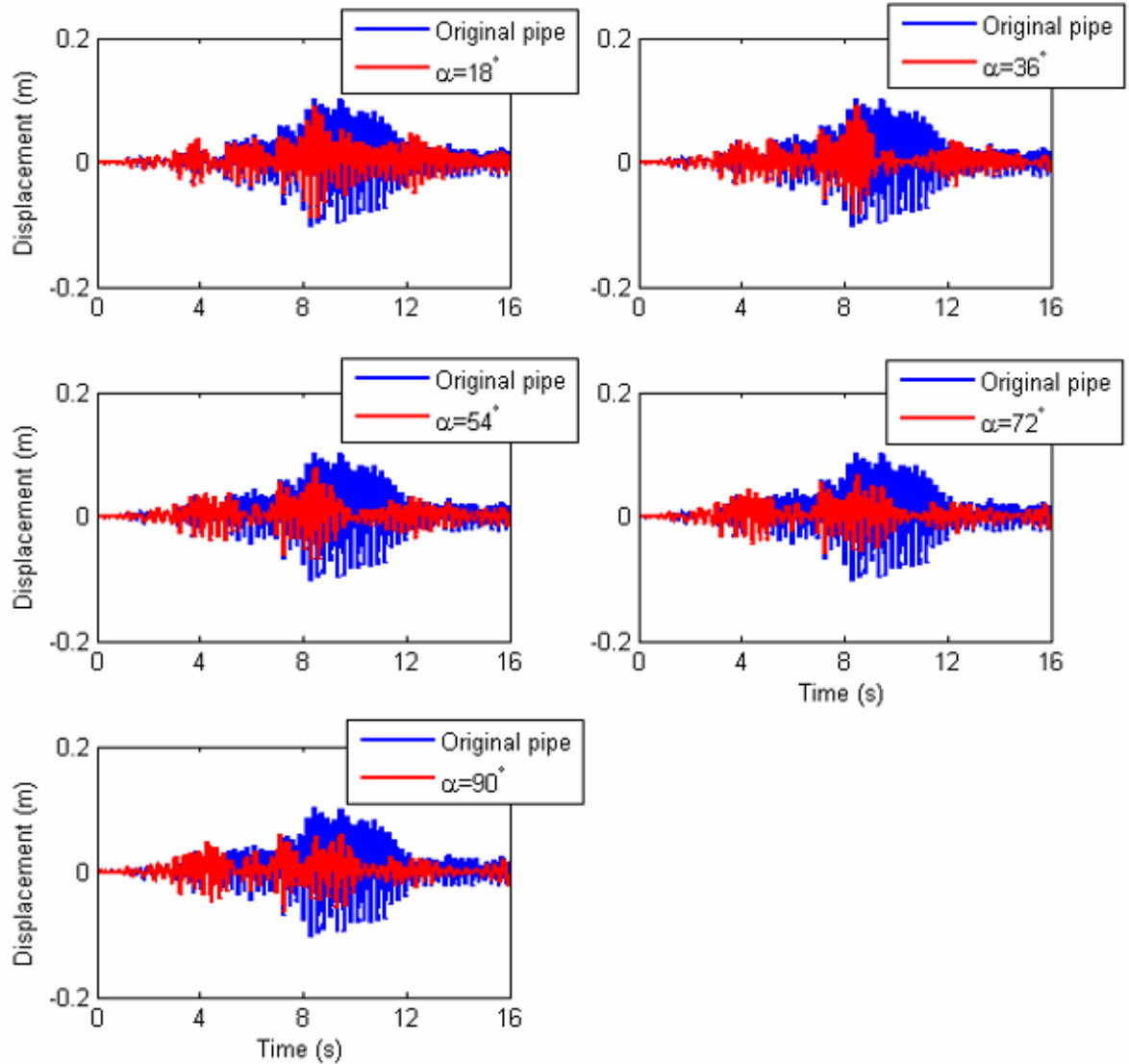

Fig. 17. Influence of constraining angle on the seismic responses of the system 
(a)

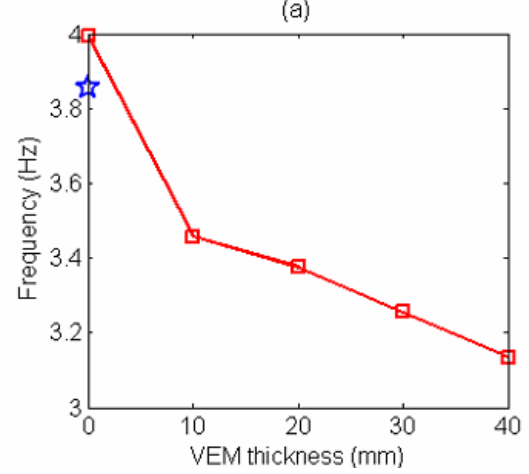

(b)

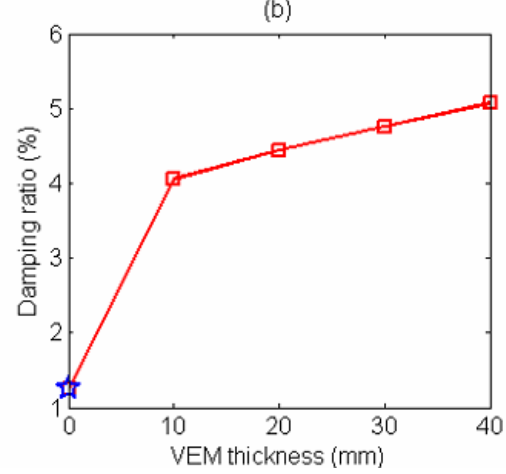

Fig. 18. Influence of VEM thickness on the modal parameters of the system: (a) vibration frequency and (b) damping ratio
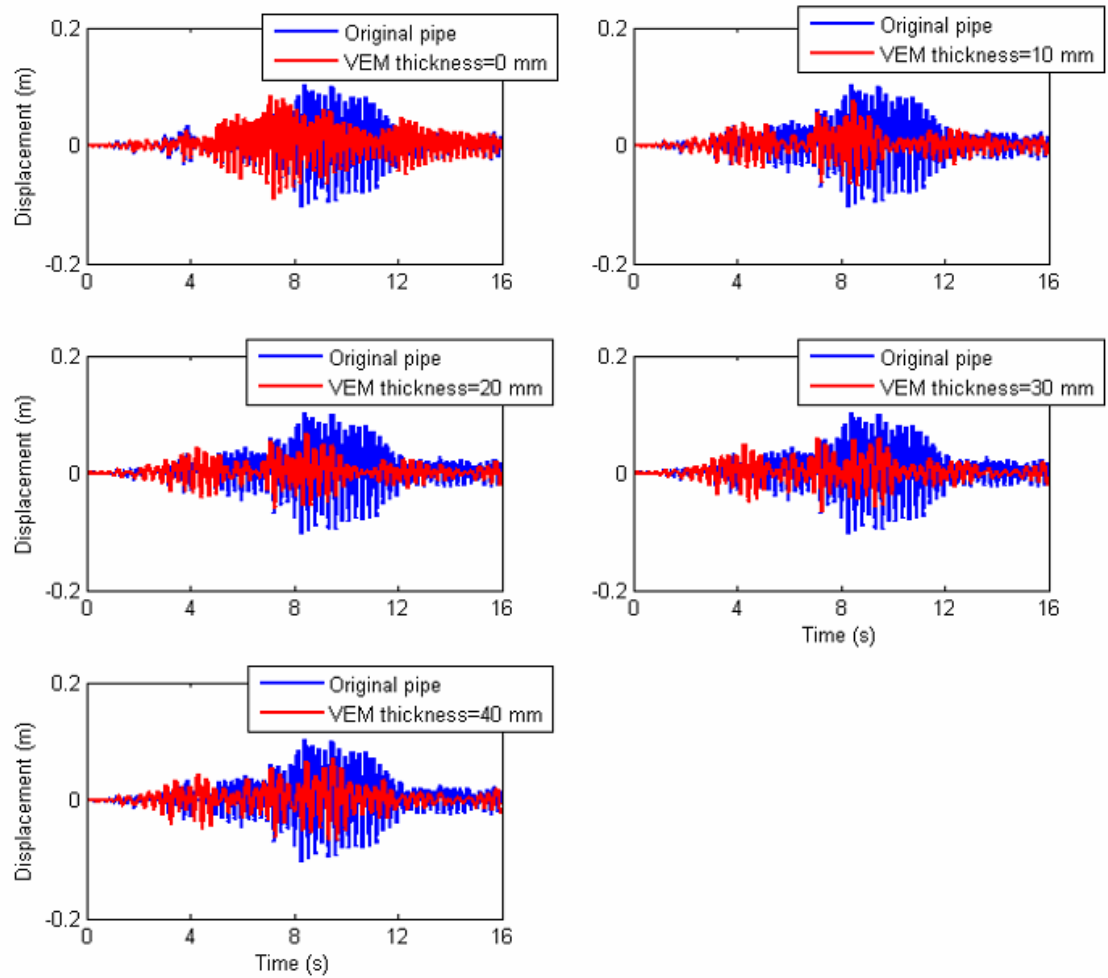

Fig. 19. Influence of VEM thickness on the seismic responses of the system 
(a)

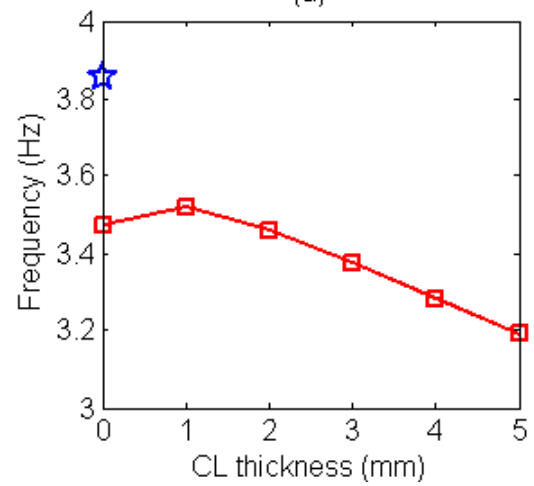

(b)

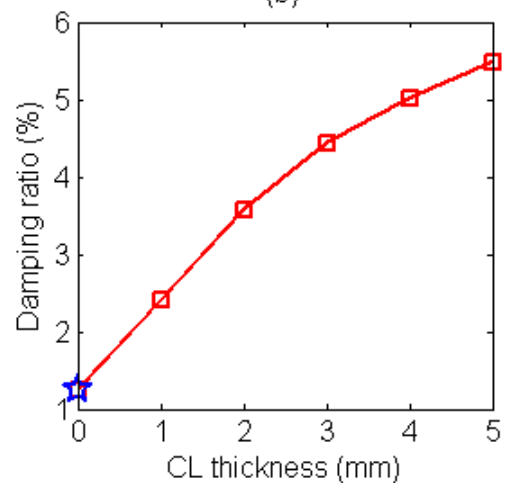

Fig. 20. Influence of CL thickness on the modal parameters of the system: (a) vibration frequency and (b) damping ratio
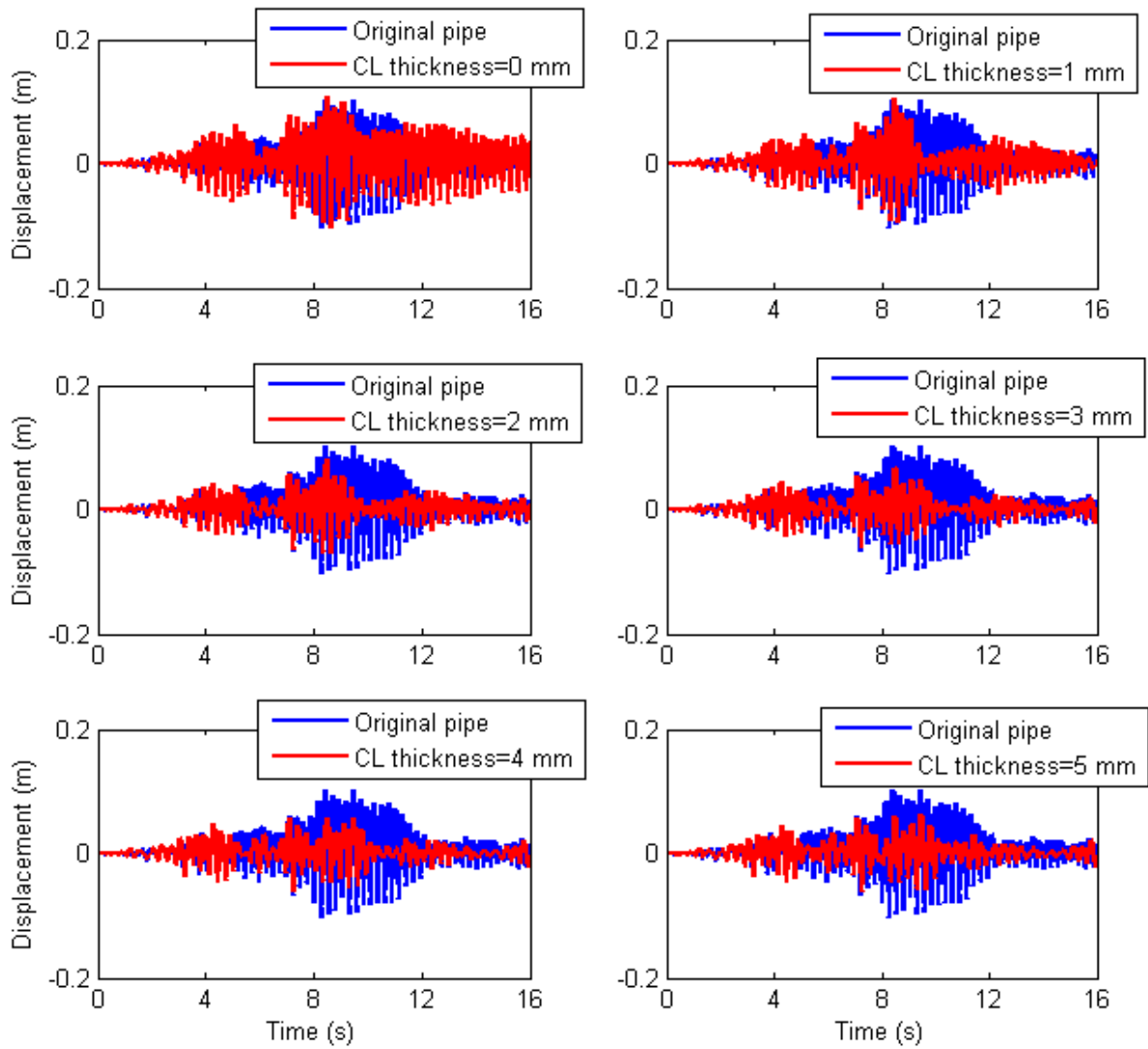

Fig. 21. Influence of CL thickness on the seismic responses of the system 
(a) Northridge earthquake

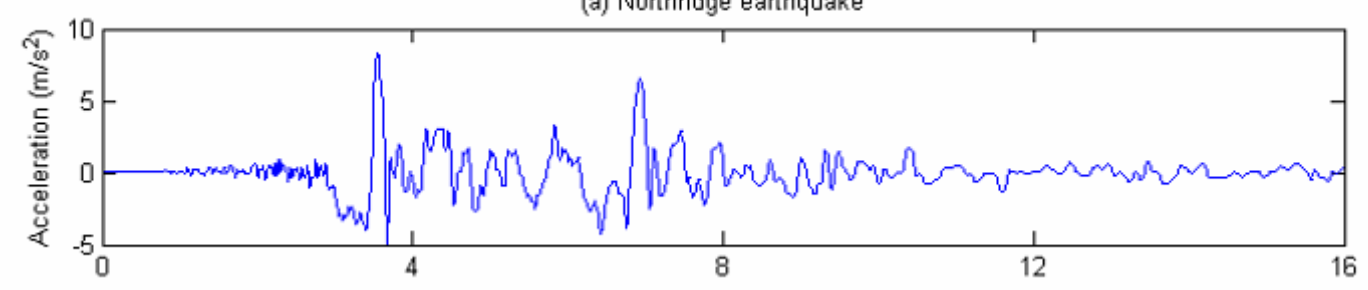

(b) San Fernando earthquake

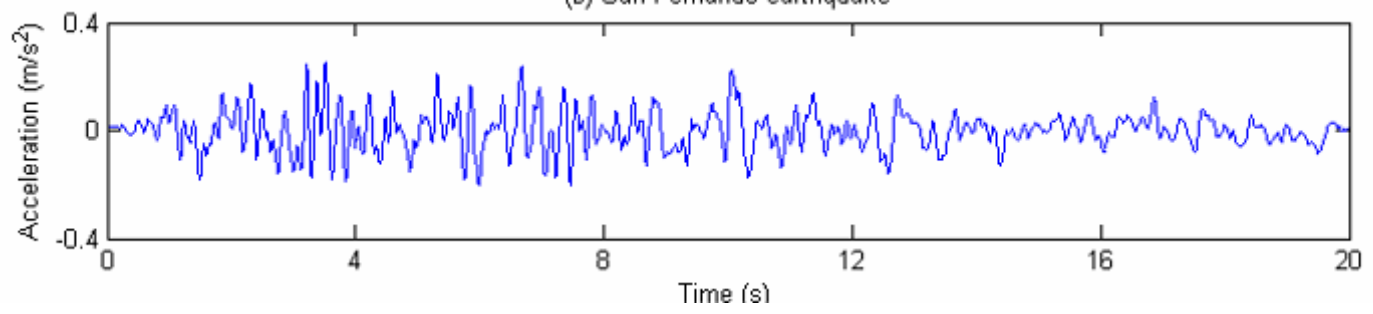

Fig. 22. Accelerograms of the selected Northridge and San Fernando earthquake loadings
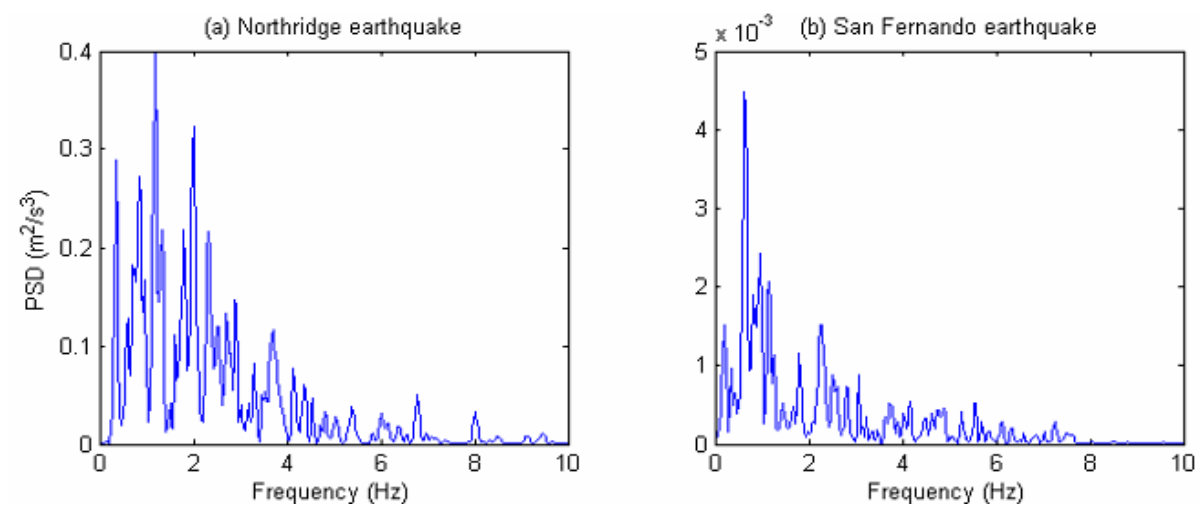

Fig. 23. PSDs of the selected Northridge and San Fernando earthquake loadings
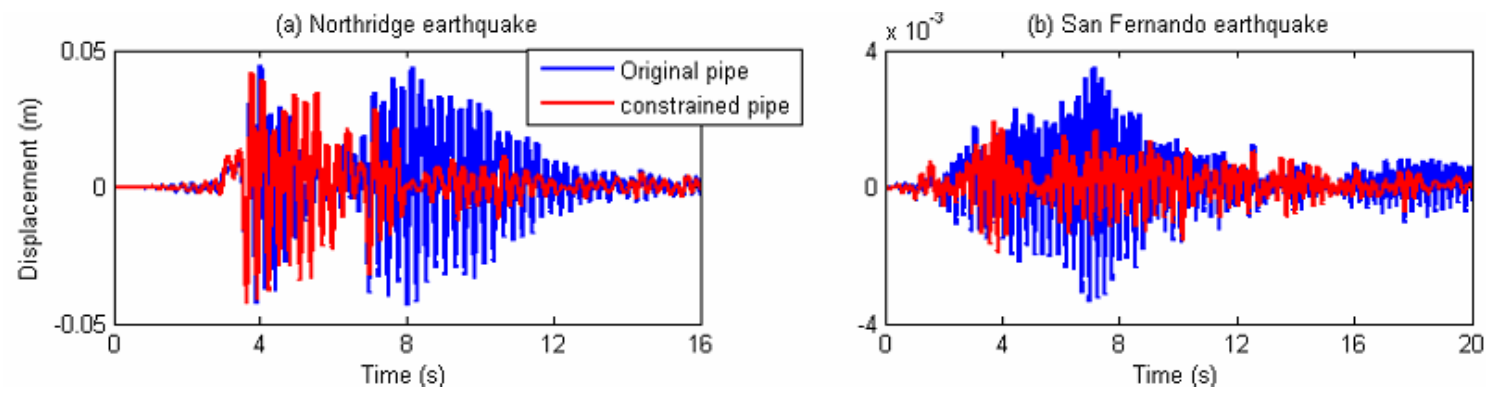

Fig. 24. Seismic responses of the original and constrained pipelines subjected to (a) Northridge and (b) San Fernando earthquakes 